NMOSD by a median of 16 years, and 11 had thymectomy. Aquaporin- 4 antibodies [AQP4-Ab] were detectable between 4 and 16 years prior to NMOSD onset. AChR-Abs decreased and the AQP4-Ab levels increased over time in concordance with MG and NMOSD, respectively. AChR-Abs were detectable at NMOSD onset in one of 2 patients diagnosed with NMOSD before MG. (Leite MI, Coutinho E, Lana-Peixoto M, et al. Myasthenia gravis and neuromyelitis optica spectrum disorder. A multicenter study of 16 patients. Neurology 2012 May 15;78:1601-1607). (Response and reprints: Dr Palace. Email: Jacqueline.palace@clneuro.ox.ac.uk).

COMMENT. The association of $\mathrm{MG}$ and NMOSD, 2 rare organ-specific autoimmune diseases mediated by 2 distinct antibodies, occurs more frequently than by chance. MG usually presents first, and respective antibodies are present years before onset of the relevant disease. The $\mathrm{MG}$ tends to be relatively mild and treatment responsive. Recent evidence suggests that the thymus is involved in both $\mathrm{MG}$ and NMOSD (Chan KH et al. J Neuroimmunol 2010;227:178-184; cited by authors). Given the risk of concurrent autoimmune diseases in patients with $\mathrm{MG}$ or NMO, routine evaluation of thyroid antibodies and AQP4-Abs may be considered in patients with early onset AChR-MG.

\title{
PROTEOMIC TECHNIQUES AS BIOMARKERS FOR MULTIPLE SCLEROSIS
}

Researchers at the Montreal Neurological Institute and other centers in Canada performed proteomics screening of CSF samples collected from 19 children at presentation of acquired inflammatory CNS demyelinating syndromes. Children were followed prospectively and 8 developed MS-defining recurrent disease activity (acquired CNS demyelinating syndrome [ADS]); 11 had no recurrent disease (ADS-monophasic) over a median period of 4.88 years (range, 2.52-6.12 years). Mass spectroscopy, peptide profiling, and quantitative immunoblotting were used to identify CSF proteins that might discriminate MS from monophasic demyelination.

Major compact myelin membrane proteins typically implicated in MS were not detected. Instead, multiple molecules that localize to the node of Ranvier and the surrounding axoglial apparatus membrane were increased by 10.2 -fold in children subsequently diagnosed with MS. The CSF proteome signature obtained at the presentation of CNS inflammation may be predictive of subsequent MS diagnosis. (Dhaunchak AS, Becker C, Schulman H, et al on behalf of the Canadian Pediatric Demyelinating Disease Group. Ann Neurol 2012 May;71:601-613). (Respond: Dr Amit Bar-Or, Neuroimmunology, Montreal Neurological Institute, McGill University, 3801 University Street, Room 111, Montreal, Quebec, H3A284, Canada. E-mail: amit.baror@mcgill.ca).

COMMENT. In the same issue of the Annals, investigators from Japan report on the use of CSF proteomic pattern analysis to discriminate MS-related disorders in 107 adult patients. (Komori $M$ et al. Ann Neurol 2012 May;71:614-623). An editorial (Bennett JL Owens GP. Ann Neurol 2012 May;71:587-588) comments that CSF proteomics is a promising window and biomarker for demyelinating disorders. 